\title{
14, 15-EET induces breast cancer cell EMT (a) CrossMark and cisplatin resistance by up-regulating integrin av 33 and activating FAK/PI3K/AKT signaling
}

Jing Luo ${ }^{1}$, Jian-Feng Yao ${ }^{3}$, Xiao-Fei Deng ${ }^{1}$, Xiao-Dan Zheng ${ }^{4}$, Min Jia², Yue-Qin Wang ${ }^{2}$, Yan Huang ${ }^{2}$ and Jian-Hua Zhu ${ }^{2^{*}}$

\begin{abstract}
Background: 14,15-epoxyeicosatrienoic acid (14,15-EET) is an important lipid signaling molecule involved in the regulation of tumor metastasis, however, the role and molecular mechanisms of 14,15-EET activity in breast cancer cell epithelial-mesenchymal transition (EMT) and drug resistance remain enigmatic.

Methods: The 14, 15-EET level in serum and in tumor or non-cancerous tissue from breast cancer patients was measured by ELISA. qRT-PCR and western blot analyses were used to examine expression of integrin av $\beta 3$. The role of 14, 15-EET in breast cancer cell adhesion, invasion was explored by adhesion and Transwell assays. The role of 14, 15-EET in breast cancer cell cisplatin resistance in vitro was determined by MTT assay. Western blot was conducted to detect the protein expressions of EMT-related markers and FAK/PI3K/AKT signaling. Xenograft models in nude mice were established to explore the roles of 14, 15-EET in breast cancer cells EMT and cisplatin resistance in vivo.

Results: In the present study, we show that serum level of 14, 15-EET increases in breast cancer patients and 14, 15-EET level of tumor tissue is higher than that of non-cancerous tissue. Moreover, 14, 15-EET increases integrin av $\beta 3$ expression, leading to FAK activation. 14, 15-EET induces breast cancer cell EMT via integrin av $\beta 3$ and FAKV PI3KJAKT cascade activation in vitro. Furthermore, we find that 14, 15-EET induces breast cancer cells EMT and cisplatin resistance in vivo, av $\beta 3$ integrin and the resulting FAK/PI3K/AKT signaling pathway are responsible for 14, 15-EET induced-breast cancer cells cisplatin resistance.
\end{abstract}

Conclusions: Our findings suggest that inhibition of 14, 15-EET or inactivation of integrin av $\beta 3 / F A K / P I 3 K / A K T$ pathway could serve as a novel approach to reverse EMT and cisplatin resistance in breast cancer cells.

Keywords: Breast cancer, 14, 15-EET, EMT, Cisplatin resistance, avß3/FAK/PI3K/AKT signaling

\section{Background}

Breast cancer is the most common malignancies worldwide. Despite recent advances in diagnosis and treatment, it remains the second leading cause of cancer-related deaths among women $[1,2]$. Chemotherapy is one of the well-established strategies of breast cancer treatment. However, drug resistance is a major cause of cancer

\footnotetext{
* Correspondence: zhujh621@163.com

2Laboratory of Clinical Immunology, Wuhan No.1 Hospital, Tongji Medical College, Huazhong University of Science and Technology (HUST), 215 Zhongshan Dadao, Wuhan, Hubei 430022, People's Republic of China

Full list of author information is available at the end of the article
}

treatment failure and cancer-related death. Therefore, it is of great clinical significance to investigate the mechanisms underlying drug resistance.

14, 15-epoxyeicosatrienoic acid $(14,15-\mathrm{EET})$ is a lipid signaling molecule which regulates various physiological processes such as proliferation, migration and inflammation $[3,4]$. Recently, it has been reported that 14, 15-EET promoted tumor cell proliferation and metastasis [5-7]. Epithelial-mesenchymal transition (EMT) is the process that epithelial cells lose polarity and cell-cell adhesion, and acquire the characteristics of mesenchymal cells $[8,9]$. Cells undergoing EMT display reduced expression of epithelial 
cell markers (such as E-cadherin, ZO-1) and increased expression of mesenchymal molecules (such as $\mathrm{N}$-cadherin, vimentin, snail, slug). EMT plays a critical role in tumor cell migration, metastasis and the acquisition of stem cell-like properties [10-13]. Although the role of 14, 15-EET in tumor invasion and metastasis has been demonstrated in recent years, the mechanism underlying the role of 14, 15EET in tumor cell EMT remains unclear.

Recently, EMT has received more and more attention for its role in cancer drug resistance. Several studies showed that the drug resistant cancer cells display features of EMT $[14,15]$. It has been found that inhibition of breast cancer cell EMT could suppress cancer drug resistance [16]. These results suggested that EMT might be associated with cancer cell drug resistance. Given that 14, 15-EET promoted tumor invasion and metastasis by inducing tumor cell EMT, the role and mechanisms of 14, 15-EET in cancer cell drug resistance still remains largely unknown.

In the present study, we found that 14,15-EET induces breast cancer cell EMT, and demonstrated that 14, 15EET up-regulates integrin $\alpha v \beta 3$ expression, which leads to the activation of FAK/PI3K/AKT signaling. Furthermore, we revealed that integrin $\alpha v \beta 3$ and FAK/PI3K/ AKT activation is required for 14, 15-EET to induce tumor cell EMT and cisplatin resistance.

\section{Methods}

\section{Patients}

The study protocol was performed according to the Declaration of Helsinki and was approved by the Ethics Committee of Wuhan No.1 Hospital of Tongji Medical College. All breast cancer patients gave their signed informed consent for the use of biological samples. Tumor tissues and noncancerous tissues were collected from 11 patients in the Wuhan No.1 Hospital of Tongji Medical College.

\section{Cell line and animals}

Human breast cancer cell MCF-7 and MDA-MB-231 were purchased from the China Center for Type Culture Collection (Wuhan, China). BALB/c athymic nude (nu/nu) mice (6-8 weeks old) were obtained from SLAC Laboratory Animal Co. Ltd. (Shanghai, China). The mice were maintained in the accredited animal facility of Tongji Medical College, and used for studies approved by the Animal Care and Use Committee of Tongji Medical College.

\section{Antibodies and reagents}

Rabbit anti-human antibodies against integrin $\alpha \mathrm{v}$ and $\beta 3$, FAK, p-FAK, PI3K, p-PI3K, AKT, p-AKT, E-cadherin, Ncadherin, vimentin, snail and LY294002 (PI3K inhibitor) were purchased from Cell Signaling (Danvers, MA, USA). slug and PF562271 were purchased from Abcam (Cambridge, MA, US). The small interfering RNAs (si RNAs) against integrin $\alpha v$, integrin $\beta 3$ and control for experiments using targeted siRNA transfection were purchased from Santa Cruz Biotechnology (Santa Cruz, CA). Lipofectamine 2000 was purchased from Invitrogen Life Technologies (Carlsbad, CA, USA). The 14, 15-EET and 14, 15-EEZE were purchased from Cayman chemical (Ann 152 Arbor, MI, USA).

\section{Cell culture}

MCF-7 and MDA-MB-231 cells were cultured in flasks in DMEM growth medium supplemented with 5\% FBS, $100 \mathrm{U} / \mathrm{ml}$ of penicillin, and $100 \mathrm{pg} / \mathrm{ml}$ of streptomycin. The cells were cultured at $37{ }^{\circ} \mathrm{C}$ in a humidified atmosphere of $95 \%$ air and $5 \% \mathrm{CO}_{2}$.

\section{Enzyme-linked immunosorbent assay (ELISA)}

A stable metabolite of 14, 15-EET, 14, 15-dihydroxyeicosatrienoic acid (14, 15-DHET) in peripheral venous blood from patients with breast cancer and healthy donors or in $\mathrm{BC}$ tissues and noncancerous tissues from breast cancer patients was measured with ELISA (14, 15-EET/DHET ELISA kit; Detroit R\&D Inc., Detroit, MI, USA) according to the manual.

\section{Measurement of cell proliferation}

Cell viability was performed using an MTT assay. Cells were added to 96 -well plates $\left(5 \times 10^{3}\right.$ cells per well) following to $24 \mathrm{~h}$ incubation. On the following day the media were removed and the cells were treated with or without 14, 15-EET and/or 14, 15-EEZE following an incubation for $72 \mathrm{~h}$. After incubation of respective time $10 \%$ of an MTT solution $(2 \mathrm{mg} / \mathrm{mL})$ was added to each well and the cells were incubated for $4 \mathrm{~h}$ at $37^{\circ} \mathrm{C}$. The formazan crystals that formed were dissolved in DMSO $(100 \mu \mathrm{L} /$ well $)$ with constant shaking for $5 \mathrm{~min}$. The absorbance of the plate was then read with a microplate reader at $540 \mathrm{~nm}$. Three replicate wells were evaluated for each analysis.

\section{Adhesion assay}

Tumor cells were added to 6-well plates $\left(5 \times 10^{5}\right.$ cells per well) which were pre-coated with fibronectin (Sigma). After $2 \mathrm{~h}$ incubation at $37^{\circ} \mathrm{C}$, non-adherent cells were harvested. Then, adherent cells were harvested by treatment with trypsin. The percentage of adherent cells was calculated. The results were expressed as A570 values. Each assay was tested in triplicate wells in three independent experiments.

\section{Matrigel invasion assay}

Cells $\left(5 \times 10^{4}\right)$ in serum-free media were seeded onto the upper chambers of modified Boyden chambers (Corning, NY, USA) in which the Transwell filter inserts were coated with Matrigel. In the lower chambers, 5\% FBS was added as a chemoattractant. After incubation for $24 \mathrm{~h}$, the membrane was washed briefly with PBS and fixed with $4 \%$ paraformaldehyde. The upper side of membrane was wiped gently with a cotton ball. The membrane was then 
stained using hematoxylin and removed. The magnitude of cells migration was evaluated by counting the migrated cells in six random clones under high-power $(\times 100)$ microscope fields. The average number of cells per field was calculated.

\section{Cell transfection}

For silencing specific gene expression, cells were treated with integrin $\alpha \mathrm{v}$ siRNA or integrin $\beta 3$ siRNA. Briefly, $5 \times 10^{5}$ MCF-7 and MDA-MB-231 cells were seeded into 6-well plate with $2 \mathrm{ml}$ antibiotic-free normal growth medium containing FBS. Transfection of integrin $\alpha v$ siRNA, integrin $\beta 3$ siRNA or control siRNA was performed according to the manufacture's protocol.

\section{Quantitative RT-PCR}

Total RNA was extracted from cells with TRIzol reagent (Invitrogen, Carlsbad, CA). Quantitative real-time PCR analyses were performed by Applied Biosystems using SYBR Premix Ex Taq ${ }^{\text {Tix }}$ (TaKaRa, Japan). The mRNA of GAPDH was used as internal control. The primers were as follows: integrin $\alpha \mathrm{v}$, sense5'-CTCGGGACTCCTGCTACCTC-3', antisense 5'-AAGAAACATCCGGGAAGACG-3' integrin $\beta 3$, sense 5'-CCGTGACGAGATTGAGTCA-3' antisense 5'-AGGATGGACTTTCCACTAGAA-3' and GAPDH, sen se 5'-TCATTGACCTCAACTACATGGTTT-3', antisense 5'-GAAGATGGTGATGGGATTTC-3'.The relative expression of $\alpha v$ and $\beta 3$ was calculated using $2^{-\Delta \Delta C t}$ method.

\section{Western blot analysis}

The whole-cell extracts were prepared using RIPA lysis buffer (Beyotime, China) with phenylmethanesulfonyl fluoride and protease inhibitor cocktail (Roche, USA). Cell lysates were separated by $8-12 \%$ SDS-PAGE and electrotransferred onto polyvinylidene difluoride membranes (Bio-Rad, USA). After being blocked using 5\% non-fat milk for $1 \mathrm{~h}$ at room temperature, membranes were incubated with the indicated primary antibodies overnight at $4{ }^{\circ} \mathrm{C}$ and probed with horseradish peroxidase-conjugated secondary antibodies (1:1000). The bands were visualized using a ChemicDocXRS system (Bio-Rad, USA).

\section{Immunohistochemistry}

Mice were inoculated i.m. in the right hind thigh with MDA-MB-231 cells $\left(2 \times 10^{6}\right)$. Tissue sections were prepared and subjected to immunohistochemical analysis. Anti-human Ki67 antibody, anti-human E-cadherin and anti-human vimentin antibody were used as primary antibodies. HRP-conjugated secondary $\mathrm{Ab}$ was used as secondary antibody. Images were obtained using an Olympus-IX71 microscope at $40 \times 10$ magnification.

For H\&E staining, the tumor tissues were embedded in paraffin according to standard histological procedures. Sections were stained with hematoxylin and eosin.

\section{Mice xenograft models}

To assay tumor cell arrest in lung during blood flow, MDA-MB-231 cells were labeled with CFSE, and injected into mice via tail vein $\left(2 \times 10^{6}\right.$ cells/mouse, $n=$ 8 for each group). Lungs of mice were harvested $5 \mathrm{~h}$ or $24 \mathrm{~h}$ after the injection. Frozen sections were prepared and analyzed by fluorescence microscopy. Fluorescent spots were counted from 20 randomly chosen fields in sections of each mouse.

Nude mice were inoculated i.m. in the right hind thigh with MDA-MB-231 cells $\left(2 \times 10^{6}\right)$. The transplanted nude mice were randomly divided into 5 groups $(n=6$ for each group). The mice were treated or untreated with14, 15-EET and/or 14, 15-EEZE (i.v. injection, $30 \mu \mathrm{g} / \mathrm{kg} / 2 \mathrm{~d}$ ). The mice were treated with cisplatin (i.p. injection, $3.0 \mathrm{mg} / \mathrm{kg} / \mathrm{d}$ ) or PBS. All mice were examined every 2 days and sacrificed 35 days after tumor inoculation. Tumor volume $(\mathrm{V})$ was monitored by measuring the length (L) and width (W) and calculated with the formula $\mathrm{V}=\left(\mathrm{L} \times \mathrm{W}^{2}\right) \times 0.5$.

\section{Statistical analysis}

The values given are means \pm S.E.M. The significance of difference between the experimental groups and controls was assessed by Student's t test. The difference was significant if the $p$ value was $<0.05$.

\section{Results}

14, 15-EET promotes breast cancer cell adhesion and migration

14, 15-EET has been reported to induce migration and invasion of human cancer cells $[5,6] .14,15$-EET is very unstable metabolites, and it's rapidly hydrolyzed by $\mathrm{sEH}$ to the more stable metabolites 14, 15-DHETs. We detected the 14, 15-DHET level in serum or in cancer and noncancerous tissues from breast cancer patients. The ELISA results showed that the levels of 14, 15DHET in serum and cancer tissues in BC patients is much higher than that of healthy donors or noncancerous tissues(Fig. 1a, b). Furthermore, we found that 14, 15-EET enhanced the adhesion ability of MCF-7 and MDA-MB-231 cells (Fig. 1c). Invasion assay showed that 14, 15-EET promoted tumor cell invasion(Fig. 1d), whereas 14, 15-EEZE, an antagonist of 14, 15-EET inhibited EET-induced cell adhesion and invasion.

We further investigated the effect of 14, 15-EET on tumor cell invasion in vivo. The fluorescent spots in lung tissues were significantly increased both $5 \mathrm{~h}$ and $24 \mathrm{~h}$ after i.v. injection of MCF-7 and MDA-MB-231 cells treated with 14, 15-EET, while 14, 15-EEZE abolished the effect of 14, 15-EET on tumor cell adhesion and invasion in vivo (Fig. 1e). These results indicated that 14, 15-EET promotes breast cancer cell adhesion and extravasation. 


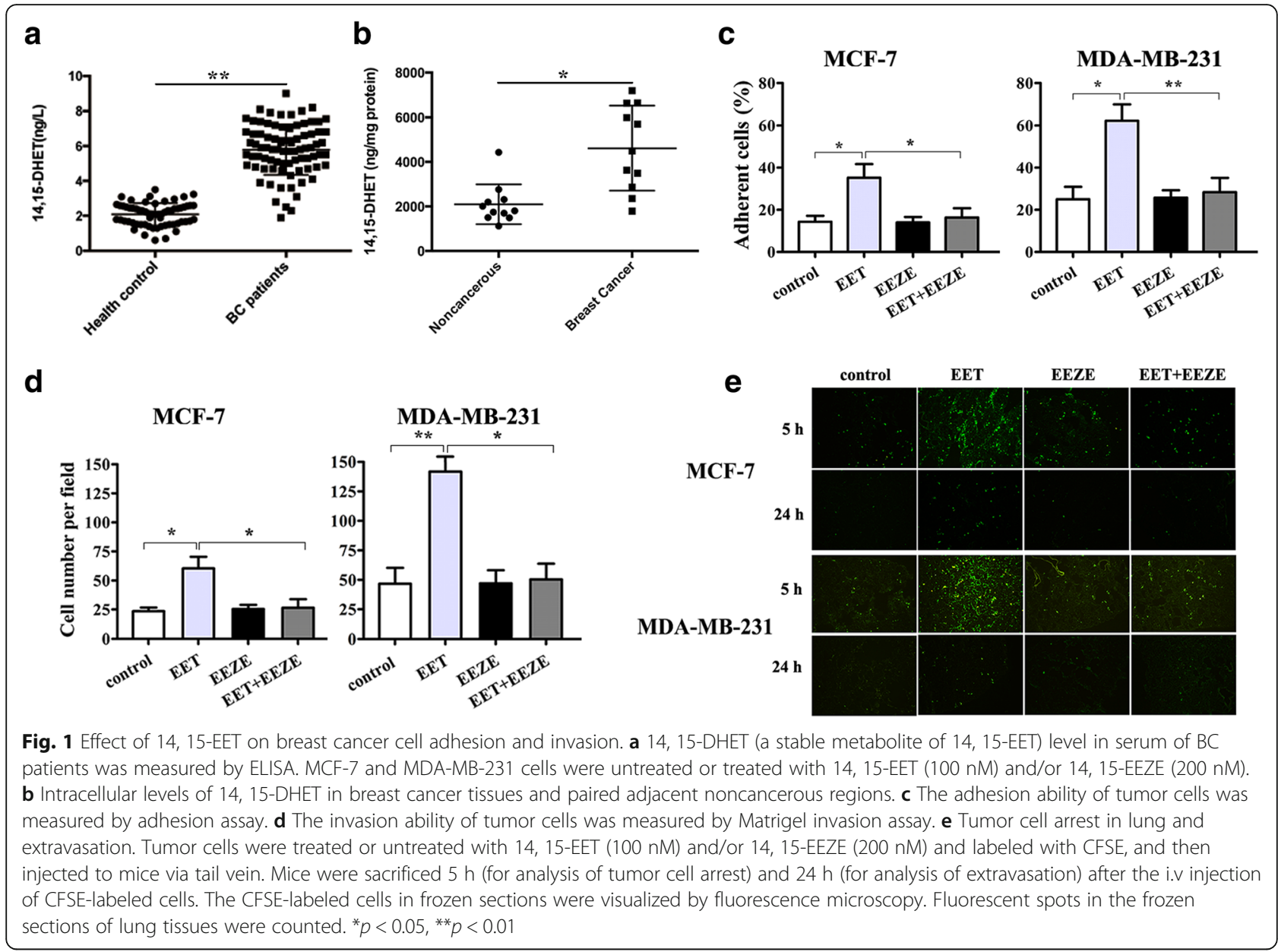

\section{4, 15-EET induces integrin av $\beta 3$ expression and FAK/ PI3K/AKT activation}

Fibronectin presents binding sites for a range of different integrins including integrin $\alpha v \beta 3$. As 14, 15-EET enhanced adhesion ability of breast cancer cells to fibronectin, we hypothesized that integrin $\alpha v \beta 3$ may be involved in $14,15-$ EET-induced breast cancer cells adhesion and invasion. We found that 14, 15-EET increased the mRNA and protein expression of $\alpha v$ - and $\beta 3$-integrin, Whereas 14, 15-EEZE, reduced EET-induced integrin $\alpha v \beta 3$ expression (Fig. 2a, b).

It is widely known that FAK is a downstream integrin $\alpha v \beta 3$ kinase. Since 14, 15-EET up-regulated integrin $\alpha v \beta 3$ expression, we investigated whether 14, 15-EET affected FAK phosphorylation. We found that 14, 15-EET increased breast cancer cells FAK phosphorylation. PI3K/AKT, downstream FAK signaling molecules were also found to be activated by 14, 15-EET, while 14, 15-EEZE inhibited 14, 15EET-induced FAK/PI3K/AKT activation (Fig. 2c).

Integrin av $\beta 3$ mediates 14, 15-EET-induced breast cancer cells migration and FAK/PI3K/AKT activation

14, 15-EET up-regulated $\alpha v \beta 3$ integrin expression and activated FAK/PI3K/AKT signaling in breast cancer cells, therefore, we investigated whether integrin $\alpha v \beta 3$ mediated the oncogenic effects of 14,15-EET. Tumor cells were transfected with integrin $\alpha \mathrm{v}$ or $\beta 3$ siRNA, the expressions of integrin $\alpha \mathrm{v}$ and $\beta 3$ were validated by western blot (Fig. 3a). Knocking down of $\alpha \mathrm{v}$ and $\beta 3$ integrin reduced 14, 15-EET-induced tumor cell FAK/PI3K/AKT phosphorylation (Fig. 3b, c) and invasion (Fig. 3d). These results indicated that 14, 15-EET promotes breast cancer cell invasion and activates FAK/PI3K/AKT signaling through up-regulating integrin $\alpha v \beta 3$ expression.

\section{Integrin av $\beta 3$ and FAK/PI3K/AKT signaling mediate 14, 15-EET-induced breast cancer cells EMT}

The above data indicated that 14, 15-EET promoted breast cancer cell invasion, given that EMT is known to be the initial step of tumor cell migration and invasion, we examined whether 14, 15-EET affect breast cancer cells EMT. When we seeded 14,15-EET-treated MCF-7 and MDA-MB-231 cells in 6-well plate, it was found that cells lost cell-cell contact and formed a scattered phenomenon (Fig. 4a). Moreover, we found that tumor cells expressed reduced E-cadherin, increased $\mathrm{N}$ cadherin, vimentin, snail and slug after treatment of 


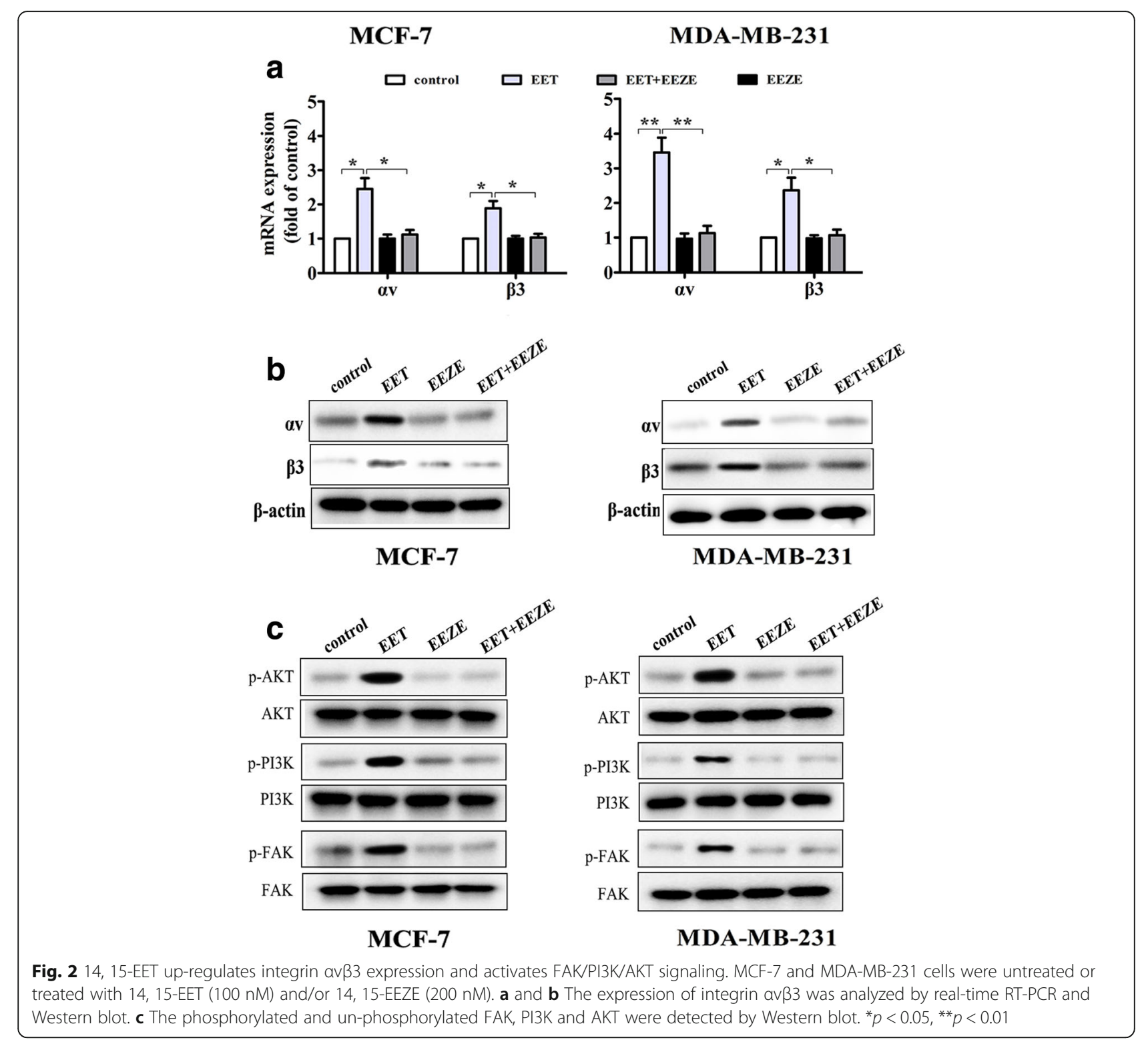

14,15-EET, while 14,15-EEZE reversed 14, 15-EETinduced EMT (Fig. 4b). We further examined whether integrin $\alpha v \beta 3$ involved in EMT induced by 14, 15-EET. As expected, knockdown of $\alpha \mathrm{v}$ or $\beta 3$ integrin inhibited 14, 15-EET-induced tumor cell EMT (Fig. 4c). PI3K signaling is responsible for EMT, to further confirm the role of FAK/PI3K/AKT signaling in 14, 15-EET-induced EMT, FAK inhibitor PF562271 and PI3K inhibitor LY294002 were utilized. We found that tumor cells treated with PF562271 or LY294002 expressed high levels of E-cadherin and low levels of $\mathrm{N}$-cadherin, vimentin, snail and slug compared with control cells (Fig. 4d). These results suggested that 14, 15-EET induces breast cancer cells EMT through $\alpha v \beta 3 / F A K /$ PI3K/AKT signaling.
14, 15-EET induces breast cancer cisplatin resistance through integrin av $\beta 3$ and FAK/PI3K/AKT signaling Tumor cells display EMT and lead to enhanced drug resistance, as 14, 15-EET induced breast cancer cells EMT, we examined the role of 14, 15-EET in tumor cells sensitivity to cisplatin. MTT assay showed that 14, 15-EET significantly reduced tumor cells sensitivity to cisplatin, while 14, 15-EEZE reversed 14, 15-EET-induced cisplatin resistance (Fig. 5a). Knocking down of $\alpha v$ or $\beta 3$ integrin reversed 14, 15-EET-induced tumor cells cisplatin resistance (Fig. 5b). Moreover, both PF562271 and LY294002 were found to reduce 14, 15-EET-induced tumor cells cisplatin resistance (Fig. 5c). These data indicated that integrin $\alpha v \beta 3$ and FAK/ PI3K/AKT signaling mediate 14, 15-EET-induced breast cancer cells cisplatin resistance. 

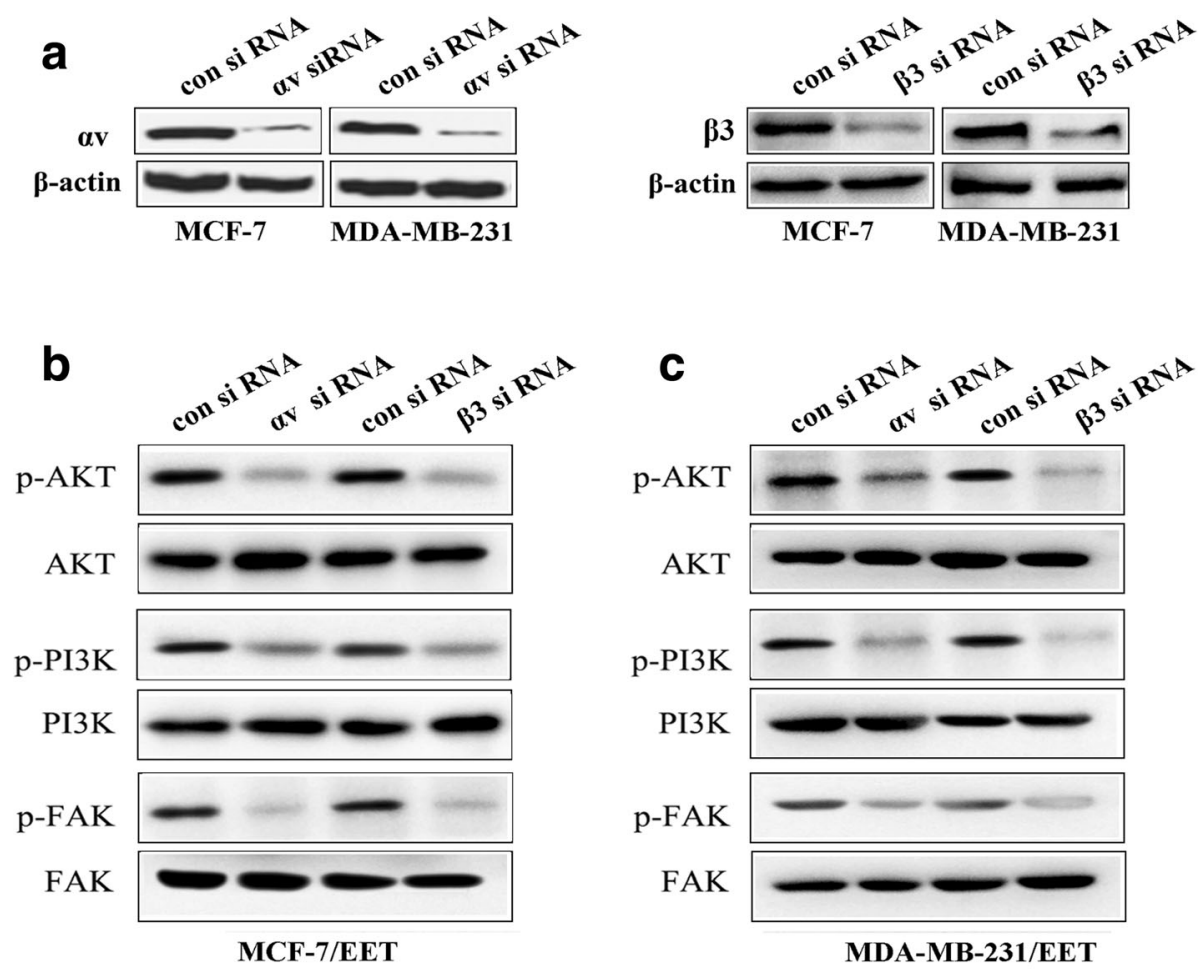

d MCF-7 MDA-MB-231
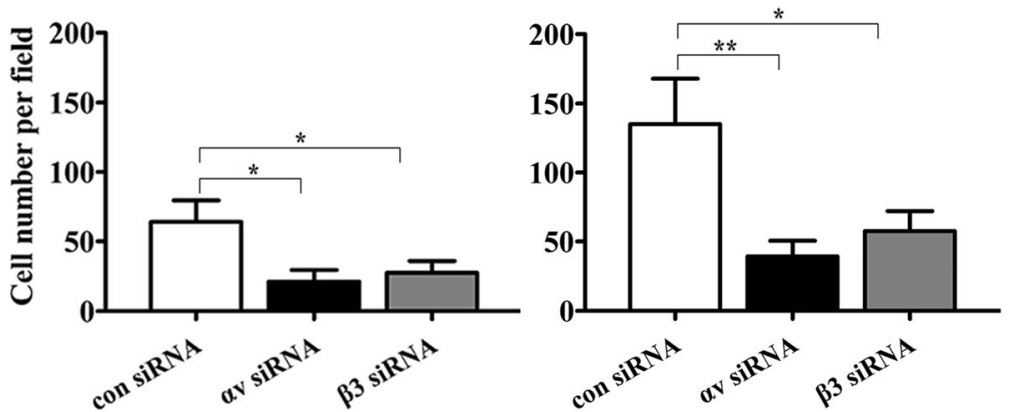

Fig. 3 14, 15-EET promotes tumor cells invasion and activates FAK/PI3K/AKT signaling through integrin av $\beta 3$. MCF-7 and MDA-MB-231 cells were transfected with integrin av or $\beta 3$ siRNA or control siRNA. a The integrin av or $\beta 3$ expression was examined by Western blot. The integrin av or $\beta 3$ knockdown tumor cells were treated with 14, 15-EET (100 nM). b and c The phosphorylated and un-phosphorylated FAK, PI3K and AKT were detected by Western blot. $\mathbf{d}$ The invasive migration assay was performed. ${ }^{*} p<0.05,{ }^{* *} p<0.01$

\section{4, 15-EET induces breast cancer cells EMT and cisplatin} resistance in vivo

We further evaluated the role of 14, 15-EET on MDA-MB231 cells EMT and cisplatin resistance in xenograft model. In line with our earlier observations, the expression of Ecadherin decreased while the expression of vimentin increased obviously after 14,15-EET treament. (Fig. 6a). The average tumor volume of cisplatin-treated tumors was significantly smaller than that of 14, 15-EET/cisplatin-treated tumors (Fig. 6b, c). Histologic examination of the tumors showed dramatically increased cellularity in the 14, 15EET/cisplatin-treated compared with cisplatin-treated tumors (Fig. 6d). Immunohistochemical staining of the 14, 15-EET/cisplatin-treated compared with cisplatin-treated tumors showed increased Ki67 levels (Fig. 6d). Whereas 14, 15-EEZE, reversed 14, 15-EET's effect. These results suggested that 14, 15-EET promotes breast cancer cells EMT and reduces cisplatin sensitivity in vivo.

\section{Discussion}

To develop a novel and efficient therapy for human breast cancer treatment, it is necessary to elucidate the molecular mechanisms underlying tumor metastasis and drug resistance. Accumulating evidence have suggested that 14, 15- 


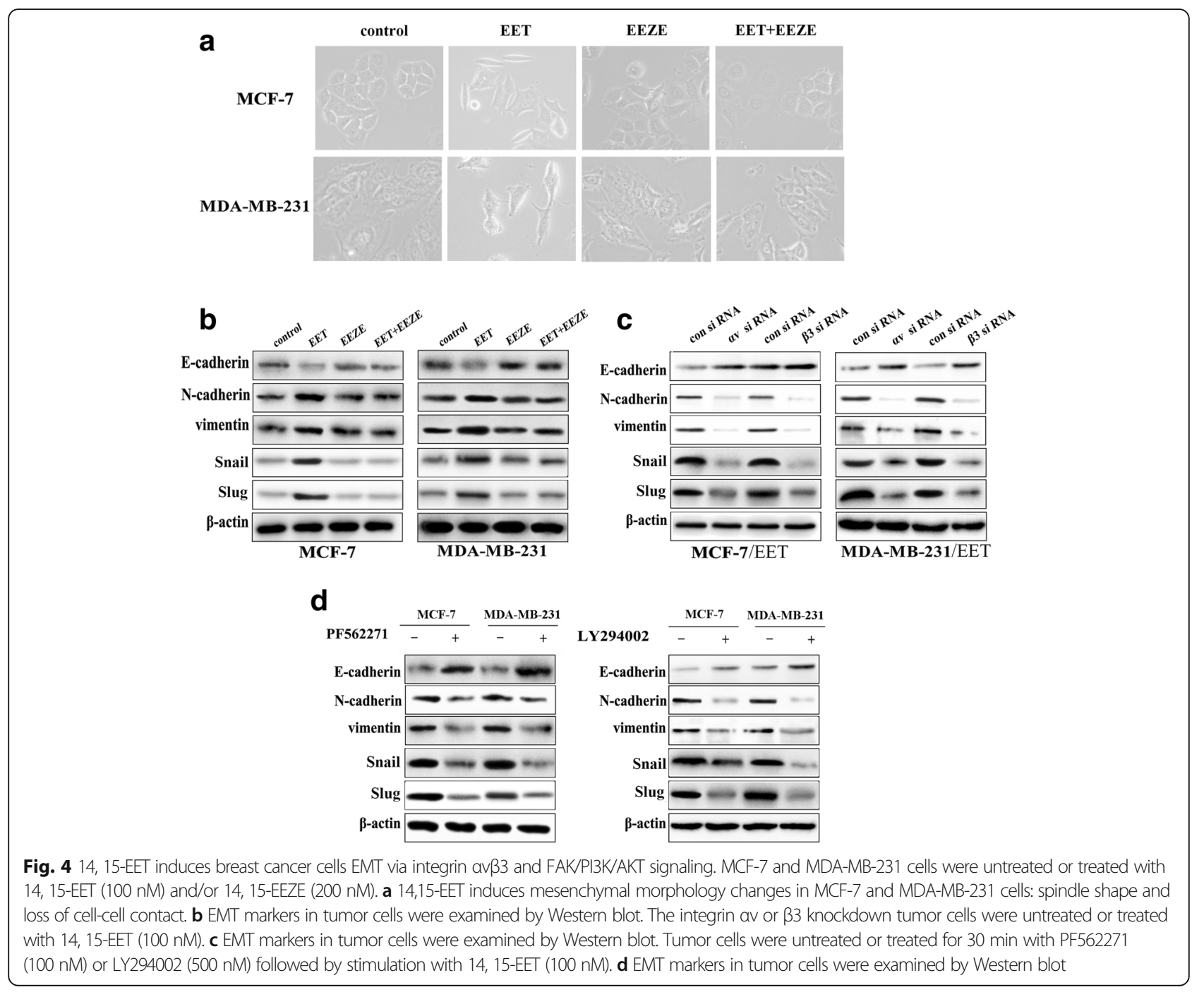

EET promotes tumor metastasis and progression in various cancers including breast cancer $[17,18]$. In the present study, we demonstrated that 14, 15-EET up-regulates integrin $\alpha v \beta 3$ expression and results in FAK/PI3K/AKT activation. Furthermore, we found that 14, 15-EET induces breast cancer cells EMT and cisplatin resistance through integrin $\alpha v \beta 3$ and its downstream FAK/PI3K/AKT/ signaling. Our finding provide an insight into the function of 14, 15 -EET in regulating breast cancer cell EMT and cisplatin resistance.

EET has been reported to enhance tumor cell motility, invasion and metastasis $[7,19]$. Our previous study found that 14, 15-EET induced neutrophils infiltration and promoted tumor metastases [17]. EMT is associated with tumor invasive and metastatic potential. However, the relationship between 14, 15-EET and breast cancer cell EMT has not been investigated. Our current study provide evidence that 14,15 EET induced breast cancer cells EMT, as demonstrated by the changed levels of EMT markers and cell morphology.
Recently, the molecular mechanisms of EMT have been extensively investigated, several signaling pathways that induce EMT have been discovered [20-22]. Integrin $\alpha v \beta 3$ has been shown to be frequently implicated in the metastasis of various tumor types [23-25]. It has been reported that integrin $\alpha v \beta 3$ is involved in tumor cell EMT [26-28]. In the current study, we found that 14, 15-EET led to a significant increase in mRNA and protein level of integrins $\alpha \mathrm{v}$ and $\beta 3$. In contrast, treatment of its antagonist 14, 15-EEZE resulted in a reversal of the 14, 15-EET effects on integrin $\alpha v \beta 3$ expression. To understand the mechanism of 14,15-EET-induced EMT, we silenced the breast cancer cells integrin $\alpha v \beta 3$. We found knockdown of integrin $\alpha v$ and $\beta 3$ reversed the effects of 14, 15-EET on the levels of EMT markers and cell morphology, these findings further confirm that integrin $\alpha v \beta 3$ mediates breast cancer cells EMT induced by 14,15-EET.

Integrin signaling is depending on the formation of adhesion complexes including FAK, after activation of FAK by 


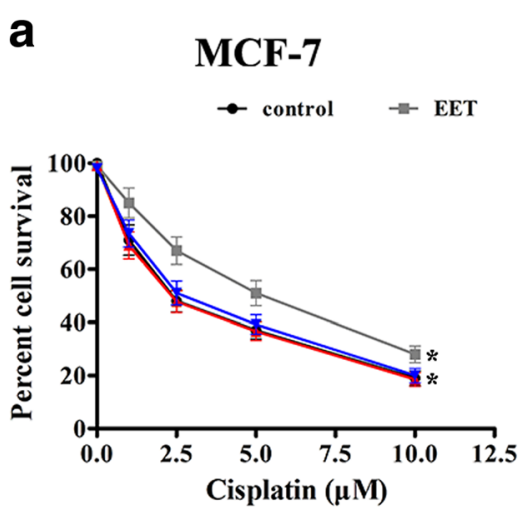

\section{MDA-MB-231}

$\rightarrow$ EEZE $\rightarrow$ EET/EEZE

b

MCF-7

MDA-MB-231
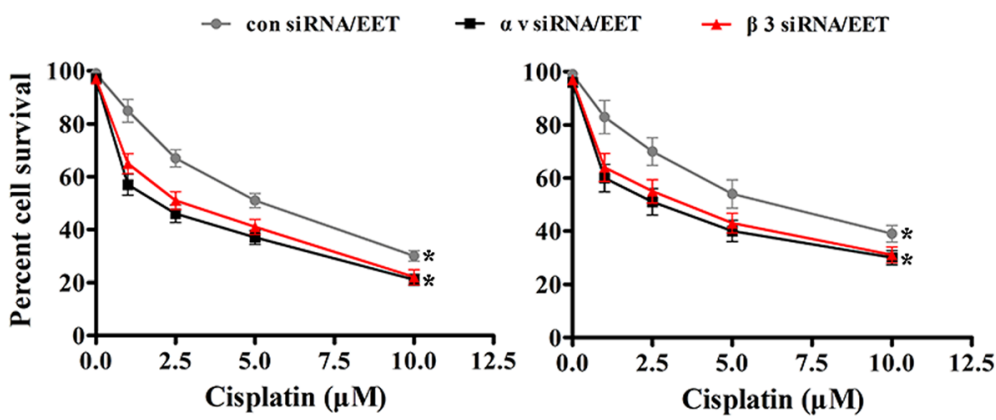

C

MCF-7

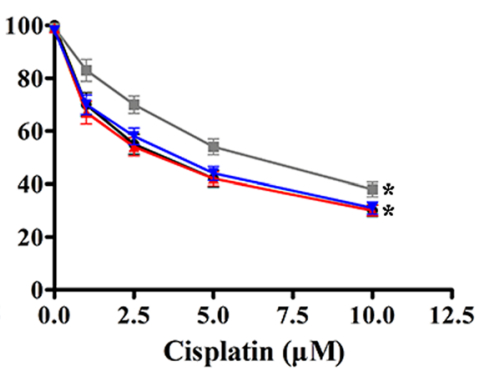

Cisplatin $(\mu \mathrm{M})$

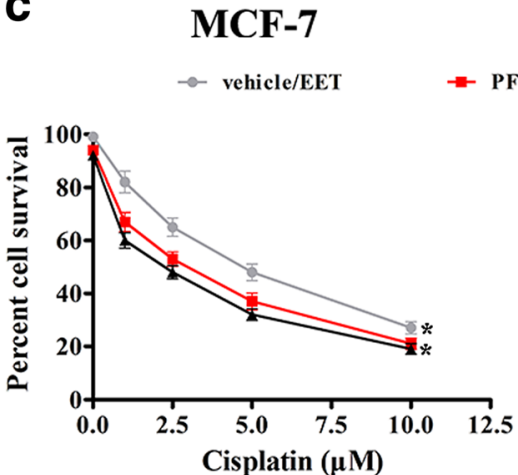

MDA-MB-231

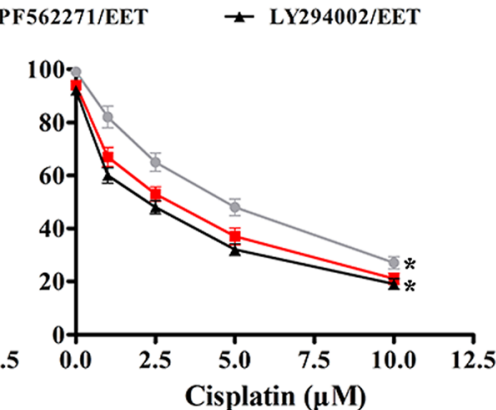

Fig. 5 14, 15-EET induces cisplatin resistance in breast cancer cells. MCF-7 and MDA-MB-231 cells were untreated or treated with 14, 15-EET (100 nM) and/or 14, 15-EEZE (200 nM). a The sensitivity of tumor cells to cisplatin was determined by MTT assay. The integrin av or $\beta 3$ knockdown tumor cells were untreated or treated with 14, 15-EET (100 nM). Tumor cells were untreated or treated with PF562271 (200 nM) or LY294002 (500 nM) followed by stimulation with 14, 15-EET (100 nM). b and $\mathbf{c}$ The sensitivity of tumor cells to cisplatin was determined by MTT assay. ${ }^{*} p<0.05$

integrins, activated FAK phosphorylates the downstream PI3K and then activates Akt [29]. Our previous study found that integrin $\alpha v \beta 3$ activated FAK and promoted tumor invasion [23]. Several studies have reported the role of FAK signaling in the induction of EMT [30, 31]. 14, 15EET has been reported to activate PI3K/AKT signaling [32]. To further elucidate the molecular mechanism of 14,15-EET-induced EMT we focused on signaling pathway implicating FAK and the downstream PI3K/AKT signaling. We demonstrated that 14, 15-EET activates breast cancer cells FAK/PI3K/AKT signaling through upregulating integrin $\alpha v \beta 3$. Furthermore, we found that inhibiting FAK by a pharmacological inhibitor of FAK, PF562271 reversed the EMT induced by 14,15-EET. Similar study was reported that FAK play a crucial role EMT through the activation of Akt signaling pathway [30]. A recent study demonstrated that FAK/PI3K/AKT signaling mediates the hepatocellular carcinoma EMT [26]. In our 


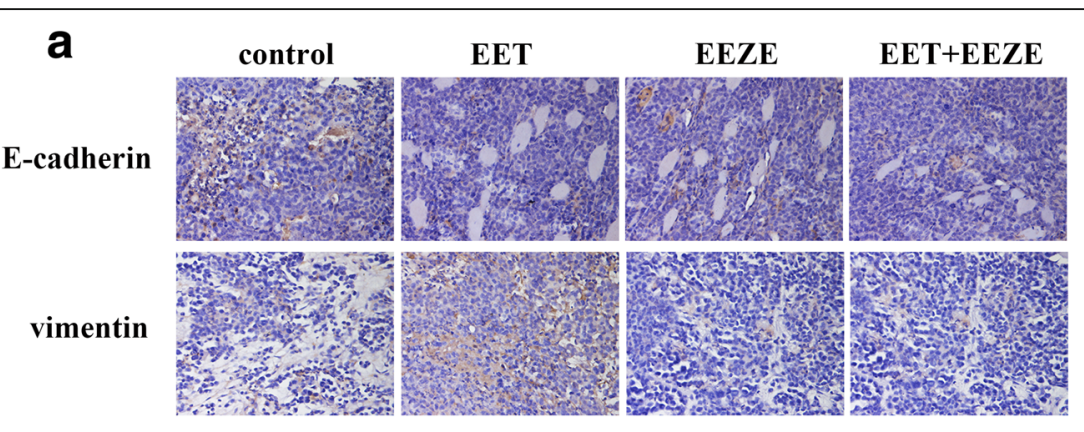

b

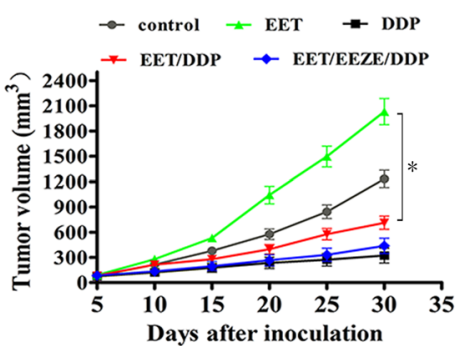

C

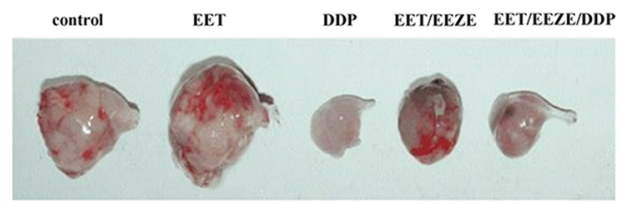

d

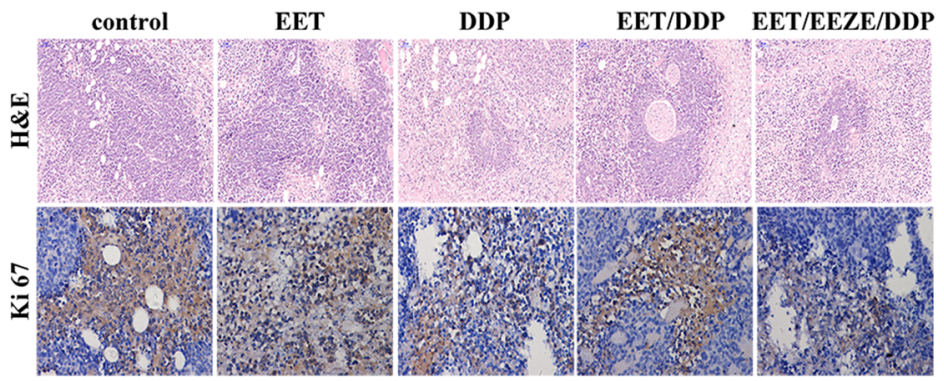

Fig. 6 14, 15-EET induces tumor cells EMT and reduces cisplatin sensitivity of breast cancer cell in vivo. Nude mice were inoculated with MDA-MB-231 cells, tumors were developed in mice followed by treatment with 14, 15-EET and $/ o r$ 14, 15-EEZE (i.v. injection, $30 \mathrm{\mu g} / \mathrm{kg} / 2 \mathrm{~d}$ ). a Representative immunohistochemical staining of EMT marker. Nude mice were inoculated with MDA-MB-231 cells, tumors were developed in mice followed by treatment with 14, 15-EET and/or 14, 15-EEZE (i.v. injection, $30 \mu \mathrm{g} / \mathrm{kg} / 2 \mathrm{~d}$ ). All mice were treated with cisplatin (i.p. injection, $3.0 \mathrm{mg} / \mathrm{kg} / \mathrm{d}$ ) or PBS. b The gross morphology of tumor samples. c The tumors volume was measured on the indicated days. $\mathbf{d}$ Tumors from mouse xenografts were removed and subjected to H\&E staining and

immunohistochemistry for Ki67. ${ }^{*} p<0.05$

study, we found that PI3K/AKT signaling inhibitor, LY294002 abrogate 14,15-EET-induced breast cancer cells EMT.

It is becoming increasingly evident that EMT is frequently accompanied with cancer drug resistance in various cancer [33-35]. Inhibition EMT reversed the drug resistance [36, 37]. It has been reported that integrin $\beta 3$ mediated tumor cell erlotinib resistance [38]. In our present study, we demonstrated that 14, 15-EET enhanced breast cancer cisplatin resistance, knockdown of integrin $\alpha v$ and $\beta 3$ abolished 14, 15-EET-induced cisplatin resistance. PI3K/AKT signaling activation may be involved in tumor cell resistance to sorafenib [26]. It has been reported that activation of AKT signaling led to drug resistance in breast cancer and ovarian cancer [39, 40]. Moreover, AKT was found to mediate the Twist2- induced cisplatin resistance [41]. Here, we found that knockdown of integrin $\alpha \mathrm{v}$ and $\beta 3$ partially inhibits FAK/ PI3K/AKT activation in 14, 15-EET-treated tumor cells. As expected, inhibition of FAK and PI3K/AKT activation by PF562271 or LY294002 substantially sensitized tumor cells to cisplatin.

As outlined above, both integrin $\alpha v \beta 3$ and FAK/PI3K/ AKT signaling have been involved in 14, 15-EETinduced breast cancer cells EMT and cisplatin resistance. Further study will be needed to refine our mechanistic model. The data on the intermediate factors connecting 14,15 -EET to integrin $\alpha v \beta 3$ is little. In addition, knockdown of integrin $\alpha \mathrm{v}$ and $\beta 3$ only partially inhibited the activation of FAK/PI3K/AKT, suggesting that additional factors downstream of 14, 15-EET may also lead to 14 , 15-EET-induced EMT and cisplatin resistance. 


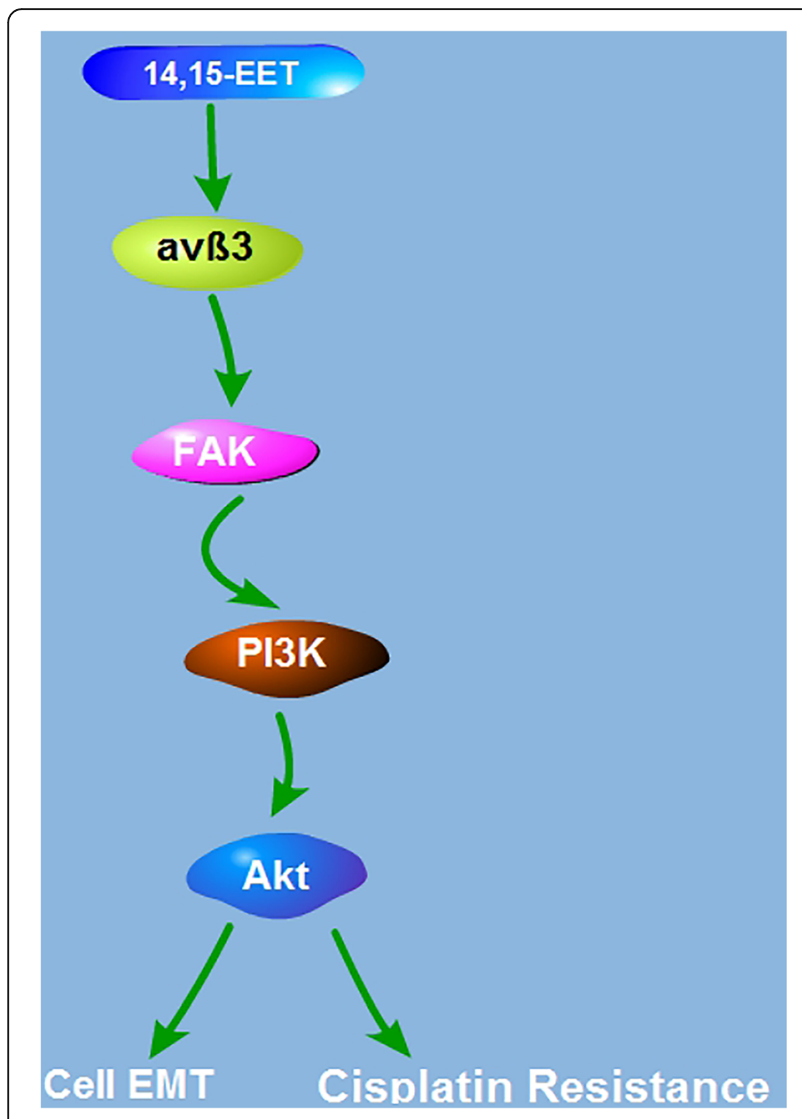

Fig. 7 Proposed schematic model for EMT and cisplatin resistance induced by 14, 15-EET in breast cancer cells. 14, 15-EET up-regulates integrin av $\beta 3$ and activates FAK/PI3K/AKT signaling, ultimately resulting in breast cancer cells EMT and cisplatin resistance

\section{Conclusions}

Taken together, we define a non-canonical function for 14, 15 -EET as an inducer of breast cancer cells EMT and drug resistance. Our results show that 14, 15-EET activates the FAK/PI3K/AKT pathway by up-regulating expression of $\alpha v \beta 3$ integrin, leading to enhanced breast cancer cells EMT and cisplatin resistance (Fig.7). Our study suggests that targeting 14, 15-EET or integrin $\alpha v \beta 3$ signaling will be able to reverse breast cancer cells EMT and cisplatin resistance.

\footnotetext{
Abbreviations

14, 15-DHET: 14, 15-dihydroxyeicosatrienoic acid; 14, 15-DHET: 14,15dihydroxyeicosatrienoic acid; 14, 15-EET: 14, 15-epoxyeicosatrienoic acid; CFSE: Carboxyfluorescein diacetate, succinimidyl ester; DMSO: Dimethyl sulfoxide; EMT: Epithelial-mesenchymal transition; FAK: Focal adhesion kinase; FN: fibronectin; Ki67: Nuclear-associated antigen; MTT: 3-(4, 5Dimethylthiazol-2-yl)-2, 5-diphenyltetrazolium bromide; PI3K: phosphatidylinositol 3 kinase; SDS-PAGE: Sodium dodecyl sulfate polyacrylamide gel electrophoresis; siRNAs: small interfering RNAs
}

\section{Funding}

This work was supported by the following funds: National Natural Science Foundation of China (81502222) and Natural Science Foundation of Hubei province (2013CFB370), China Postdoctoral Science Foundation (2016 M602311).

\section{Availability of data and materials}

Data sharing not applicable to this article as no datasets were generated oranalyzed during the current study.

\section{Authors' contributions}

$\mathrm{Z}-J \mathrm{H}$ and $\mathrm{L} J$ conceived the concept, designed the experiments and wrote the manuscript. $L, Y$-JF, D-XF, Z-XD carried out and interpreted the experiments. W-YQ established the animal models. JM and HY provided clinical samples and carried out the patient sample analyses. Z-JH, LJ, Y-JF and W-YQ analyzed the data. All authors read and approved the final manuscript.

\section{Ethics approval and consent to participate}

All experimental procedures were approved by the Institutional Review Board of the Huazhong University of Science and Technology. Written informed consent was obtained for all patient samples. The biological samples were collected from patients with breast cancer at Wuhan No.1 Hospital (during 2014-2015). The control samples were obtained from the healthy volunteers. The study protocol was performed according to the Declaration of Helsinki and was approved by the ethics committees at the Wuhan No.1 Hospital.

\section{Consent for publication}

Not applicable.

\section{Competing interests}

The authors declare that they have no competing interests.

\section{Publisher's note}

Springer Nature remains neutral with regard to jurisdictional claims in published maps and institutional affiliations.

\section{Author details}

${ }^{1}$ Department of Immunology, Tongji Medical College, Huazhong University of Science and Technology (HUST), Wuhan, Hubei, People's Republic of China. 'Laboratory of Clinical Immunology, Wuhan No.1 Hospital, Tongji Medical College, Huazhong University of Science and Technology (HUST), 215 Zhongshan Dadao, Wuhan, Hubei 430022, People's Republic of China. ${ }^{3}$ Quanzhou Maternal and Child Health Care Hospital, Quanzhou, People's Republic of China. ${ }^{4}$ Department of Radiology, Tongji Hospital, Tongji Medical College, Huazhong University of Science and Technology (HUST), Wuhan, Hubei, People's Republic of China.

Received: 25 October 2017 Accepted: 31 January 2018

Published online: 09 February 2018

\section{References}

1. Lv W, Chen N, Lin Y, Ma H, Ruan Y, Li Z, et al. Macrophage migration inhibitory factor promotes breast cancer metastasis via activation of HMGB1/TLR4/NF kappa B axis. Cancer Lett. 2016;375:245-55.

2. Siegel R, Naishadham D, Cancer JA. Statistics. CA Cancer J Clin. 2013;63:11-30.

3. Spector AA, Norris AW. Action of epoxyeicosatrienoic acids on cellular function. Am J Physiol Cell Physiol. 2007;292:C996-1012.

4. Fleming I. Vascular cytochrome p450 enzymes: physiology and pathophysiology. Trends Cardiovasc Med. 2008;18:20-5.

5. Panigrahy D, Edin ML, Lee CR, Huang $S$, Bielenberg DR, Butterfeld CE, et al. Epoxyeicosanoids stimulate multiorgan metastasis and tumor dormancy escape in mice. J Clin Invest. 2012;122:178-91.

6. Wei X, Zhang D, Dou X, Niu N, Huang W, Bai J, et al. Elevated 14,15epoxyeicosatrienoic acid by increasing of cytochrome P450 2C8, 2C9 and $2 \mathrm{~J} 2$ and decreasing of soluble epoxide hydrolase associated with aggressiveness of human breast cancer. BMC Cancer. 2014;14:841.

7. Jiang JG, Ning YG, Chen C, Ma D, Liu Z, Yang S, et al. Cytochrome p450 epoxygenase promotes human cancer metastasis. Cancer Res. 2007;67:6665-74.

8. Kajiyama H, Shibata K, Terauchi M, Yamashita M, Ino K, Nawa A, et al. Chemoresistance to paclitaxel induces epithelial-mesenchymal transition and enhances metastatic potential for epithelial ovarian carcinoma cells. Int J Oncol. 2007;31(2):277-83. 
9. Sun L, Yao Y, Liu B, Lin Z, Lin L, Yang M, et al. MiR-200b and miR-15b regulate chemotherapy-induced epithelial mesenchymal transition in human tongue cancer cells by targeting BMI1. Oncogene. 2012;31:432-45.

10. Ewald AJ, Brenot A, Duong M, Chan BS, Werb Z. Collective epithelial migration and cell rearrangements drive mammary branching morphogenesis. Dev Cell. 2008;14:570-81.

11. Thiery JP, Acloque H, Huang RY, Nieto MA. Epithelial-mesenchymal transitions in development and disease. Cell. 2009;139:871-90.

12. López-Novoa JM, Nieto MA. Inflammation and EMT: an alliance towards organ fibrosis and cancer progression. EMBO Mol Med. 2009;1:303-14.

13. Keshamouni VG, Schiemann WPEMT. In tumor metastasis: a method to the madness. Future Oncol. 2009;8:1109-11.

14. Chen QY, Jiao DM, Wang J, Hu H, Tang X, Chen J, et al. miR-206 regulates cisplatin resistance and EMT in human lung adenocarcinoma cells partly by targeting MET. Oncotarget. 2016;26:24510-26.

15. Huang D, Duan $H$, Huang $H$, Tong $X, \operatorname{Han} Y, R u G$, et al. Cisplatin resistance in gastric cancer cells is associated with HER2 upregulation-induced epithelialmesenchymal transition. Sci Rep. 2016;6:20502.

16. Raza U, Saatci Ö, Uhlmann S, Ansari SA, Eyüpoğlu E, Yurdusev E, et al. The miR-644a/CTBP1/p53 axis suppresses drug resistance by simultaneous inhibition of cell survival and epithelialmesenchymal transition in breast cancer. Oncotarget. 2016;7:49859-77.

17. Luo J, Feng $X X$, Luo C, Wang $Y$, Li D, Shu $Y$, et al. 14, 15-EET induces the infiltration and tumor-promoting function of neutrophils to trigger the growth of minimal dormant metastases. Oncotarget. 2016;7:43324-36.

18. Nithipatikom K, Brody DM, Tang AT, Manthati VL, Falck JR, Williams CL, et al. Inhibition of carcinoma cell motility by epoxyeicosatrienoic acid (EET) antagonists. Cancer Sci. 2010;101:2629-36.

19. Wang D, Dubois RN. Epoxyeicosatrienoic acids: a double-edged sword in cardiovascular diseases and cancer. J Clin Invest. 2012;122(1):19-22.

20. Thiery JP, Sleeman JP. Complex networks orchestrate epithelialmesenchymal transitions. Nat Rev Mol Cell Biol. 2006;7:131-42.

21. Huber MA, Azoitei N, Baumann B, Grünert S, Sommer A, Pehamberger H, et al. NF-kappaB is essential for epithelial-mesenchymal transition and metastasis in a model of breast cancer progression. J Clin Invest. 2004;114:569-81.

22. Lamouille S, Xu J, Derynck R. Molecular mechanisms of epithelialmesenchymal transition. Nat Rev Mol Cell Biol. 2014;15:178-96.

23. Zhu J, Luo J, Li Y, Jia M, Wang Y, Huang $Y$, et al. HMGB1 induces human nonsmall cell lung cancer cell motility by activating integrin av $\beta 3 / F A K$ throughTLR4/NF-kB signaling pathway. Biochem Biophys Res Commun. 2016:480:522-7.

24. Heyder C, Gloria-Maercker E, Hatzmann W, Niggemann B, Zänker KS, Dittmar T. Role of the beta1-integrin subunit in the adhesion, extravasation and migration of T24 human bladder carcinoma cells. Clin Exp Metastasis. 2005;22:99-106.

25. Seales EC, Jurado GA, Brunson BA, Wakefield JK, Frost AR, Bellis SL, et al. Hypersialylation of beta1 integrins, observed in colon adenocarcinoma, may contribute to cancer progression by up-regulating cell motility. Cancer Res. 2005;65:4645-52.

26. Zhang PF, Li KS, Shen YH, Gao PT, Dong ZR, Cai JB, et al. Galectin-1 induces hepatocellular carcinoma EMT and sorafenib resistance by activating FAK PI3KJAKT signaling. Cell Death Dis. 2016;7:e2201.

27. Mori S, Kodaira M, Ito A, Okazaki M, Kawaguchi N, Hamada Y. Enhanced Expression of Integrin av $\beta 3$ Induced by TGF- $\beta$ Is Required for the Enhancing Effect of Fibroblast Growth Factor 1 (FGF1) in TGF- $\beta$-Induced Epithelial-Mesenchymal Transition (EMT) in Mammary Epithelial Cells. PLoS One. 2015;10:e0137486

28. Shah PP, Fong MY, Kakar SS. PTTG induces EMT through integrin aVB3-focal adhesion kinase signaling in lung cancer cells. Oncogene. 2012;31:3124-35.

29. Oudart JB, Doué M, Vautrin A, Brassart B, Sellier C, Dupont-Deshorgue A, et al. The anti-tumor NC1 domain of collagen XIX inhibits the FAK/PI3K/Akt/ mTOR signaling pathway through av 33 integrin interaction. Oncotarget. 2016:12:1516-28.

30. Deng B, Yang X, Liu J, He F, Zhu Z, Zhang C. Focal adhesion kinase mediates TGF-beta1-induced renal tubular epithelial-tomesenchymaltransition in vitro. Mol Cell Biochem. 2010;340:21-9.

31. Wendt MK, Smith JA, Schiemann WP. Transforming growth factor-betainduced epithelialmesenchymal transition facilitates epidermal growth factor-dependent breast cancer progression. Oncogene. 2010;29:6485-98.

32. Zhang B, Cao H, Rao GN. Fibroblast growth Factor-2 is a downstream mediator of phosphatidylinositol 3-kinase-Akt signaling in 14, 15-Epoxyeicosatrienoic acid-induced angiogenesis. J Biol Chem. 2006;281:905-14.
33. Huang J, Li H, Ren G. Epithelial-mesenchymal transition and drug resistance in breast cancer (review). Int J Oncol. 2015:47(3):840-8.

34. McConkey DJ, Choi W, Marquis L, Martin F, Williams MB, Shah J, et al. Role of epithelial-to-mesenchymal transition (EMT) in drug sensitivity and metastasis in bladder cancer. Cancer Metastasis Rev. 2009;28:335-44.

35. Arumugam T, Ramachandran V, Fournier KF, Wang HM, Marquis L, et al. Epithelial to mesenchymal transition contributes to drug resistance in pancreatic cancer. Cancer Res. 2009;69:5820-8.

36. Shang $Y$, Cai $X$, Fan D. Roles of epithelial-mesenchymal transition in cancer drug resistance. Curr Cancer Drug Targets. 2013;13:915-29.

37. Wang Z, Li Y, Ahmad A, Azmi AS, Kong D, Banerjee S, et al. Targeting miRNAs involved in cancer stem cell and EMT regulation: an emerging concept in overcoming drug resistance. Drug Resist Updat. 2010;13(4-5):109-18.

38. Seguin L, Kato S, Franovic A, Camargo MF, Lesperance J, Elliott KC, et al. An integrin 33 -KRAS-RalB complex drives tumor stemness and resistance to EGFR inhibition. Nat Cell Biol. 2014;16:457-68.

39. Cheng GZ, Chan J, Wang Q, Zhang W, Sun CD, Wang LH. Twist transcriptionally up-regulates AKT2 in breast cancer cells leading to increased migration, invasion, and resistance to paclitaxel. Cancer Res. 2007;67:1979-87.

40. Roberts CM, Tran MA, Pitruzzello MC, Wen W, Loeza J, Dellinger TH, et al. TWIST1 drives cisplatin resistance and cell survival in an ovarian cancer model, via upregulation of GAS6, L1CAM, and Akt signaling. Sci Rep. 2016:6:37652.

41. Wang T, Li Y, Tuerhanjiang A, Wang W, Wu Z, Yuan M, et al. Twist2 contributes to cisplatin-resistance of ovarian cancer through the AKT/GSK3beta signaling pathway. Oncol Lett. 2014;7(4):1102-8.

\section{Submit your next manuscript to BioMed Central and we will help you at every step:}

- We accept pre-submission inquiries

- Our selector tool helps you to find the most relevant journal

- We provide round the clock customer support

- Convenient online submission

- Thorough peer review

- Inclusion in PubMed and all major indexing services

- Maximum visibility for your research

Submit your manuscript at www.biomedcentral.com/submit
) Biomed Central 